\title{
Prolonged Use of n95 Mask a Boon or Bane to Healthcare Workers During Covid-19 Pandemic
}

\author{
Kumar Shubhanshu' ${ }^{1}$ Avaneesh Singh ${ }^{1}$
}

Received: 30 August 2020/Accepted: 11 January 2021/Published online: 25 January 2021

(C) Association of Otolaryngologists of India 2021

\begin{abstract}
The novel coronavirus, referred to as SARSCOV 2, originated in Wuhan, China in December 2019. Since many Health Care Workers (HCW) and general public lost their lives. The only thing which can prevent from being infected is social distancing and wearing of mask (N95) and wearing of mask has its own adverse effects. This is a retrospective study conducted in the Department of Otorhinolaryngology, Hind Institute of Medical Sciences, Sitapur from April 2020 to August 2020. The most common symptoms of wearing mask were headache, nasal dryness, eye dryness and acne for which extra precautions to be taken to deal with these conditions during COVID-19 pandemic. HCW should wear mask to prevent from exposing but while using the mask there are certain prerequisites which has to be followed to prevent from not only thr COVD - 19 but also the complications of prolonged use of N95 mask.
\end{abstract}

Keywords COVID-19 $\cdot$ N95 mask $\cdot$ HCW

\section{Introduction}

The novel coronavirus, referred to as SARS-COV 2, originated in Wuhan, China in December 2019. Within a short amount of time, hundreds of thousands of cases were diagnosed around the world, causing the World Health Organization to announce it as an infectious disease pandemic on January and COVID-19 is spread by respiratory

Kumar Shubhanshu

shubhanshu0304@gmail.com

1 Department of Otorhinolaryngology, HIMS, Ataria, Sitapur, U.P, India droplets, and healthcare professionals are mandated to wear PPE when caring for COVID-19 patients. PPE includes gowns, gloves, masks and face shields. Facemasks and respirators are important components of personal protective equipment for health care workers in hospitals and public civilians during COVID-19 pandemic. The importance of facemasks and respirators is that they filter fine airborne particles from reaching the respiratory system and prevent inter-individual infection [1].

The CDC and WHO recommend wearing N95 masks during care of patients with highly transmissible diseases such as tuberculosis, SARS, and COVID-19. The N in N95 stands for NIOSH, the National Institute for Occupational Safety and Health of the United States and 95 indicates filter efficiency of particles. Thus, an N95 mask is 95\% effective at filtering airborne particles including very small ones. In comparison, while surgical masks provide a barrier against large respiratory particles, they are ineffective at providing protection from smaller particles. Surgical masks also do not prevent leakage around the mask when the user inhales. Therefore, surgical masks are ineffective and do not provide enough protection when performing direct care for patients with COVID-19 [2].

The last time when prolonged use of PPE among healthcare professionals was during the SARS (severe acute respiratory syndrome) outbreak in 2003-2004 which originated in Guangdong, China. Studies focusing on effects of prolonged use of PPE during the SARS outbreak were published in subsequent years $[1,3]$

Wearing masks for a prolonged amount of time can causes physiologic and psychologic burdens and can decrease work efficiency. Activity cannot be performed as long or as efficiently while wearing masks as compared to when masks are not worn. Additionally, the timeframe that an activity can be sustained is decreased when wearing 
masks and PPE. Prolonged use of mask can lead to headache, nasal dryness, epistaxis, dryness of eyes, adverse skin reactions such as rashes, acne, and itching from mask use [4]

\section{Materials and Methods}

The digital survey was done in the form of questions based on conditions among the 300 health care workers including doctors, paramedical staff, pharmacist, etc. from the beginning of April 2020 to August 2020 to the healthcare professionals during COVID-19 pandemic. The survey was done in the Hind Institute Of Medical Sciences, Sitapur Uttar Pradesh in the department of Otorhinolaryngology and Ophthalmology. The population below 18 years of age and above 50 years of age were excluded from the survey.

\section{Results}

Among the 423 participants of this survey, 283 (67\%) primarily wear a N95 mask and $140(33 \%)$ (Table 1).

wear a surgical mask throughout their shift (Table 2). Out of 423 HCW 103 (24\%) were females and 320 (76\%) were males (Table 3). The 21 year was the lowest age among all and 47 year was the oldest one among them. Headache (23\%), Nasal Dryness (22\%), Eye Dryness (19\%), Acne (12\%) were the most common symptoms among the all followed by other minor symptoms including Skin Breakdown (5\%), Impaired Cognition (5\%) and Epistaxis (5\%), and all symptoms were related to the time duration of wearing the mask. (9\%) of HCW doesn't have any type of symptoms (Table 4). Most of the HCW don't have the prior history of any such conditions.

Headache which was the most common symptom resolve after taking medications or removal of the mask. For Nasal Dryness HCW use saline nasal drops or gel to keep the nasal mucosa moist prior wearing mask because in few HCW it leads to minor epistaxis due to crusting. For Eye Dryness HCW use eye lubricating drops during wear of mask and in between also and it was most commonly

Table 1 Age

\begin{tabular}{lll}
\hline AGE & Number & Percentage \\
\hline $18-26$ & $\mathbf{9 6}$ & $\mathbf{2 3 \%}$ \\
$27-34$ & $\mathbf{1 2 1}$ & $\mathbf{2 9 \%}$ \\
$35-42$ & $\mathbf{9 3}$ & $\mathbf{2 2 \%}$ \\
$43-50$ & $\mathbf{1 1 3}$ & $\mathbf{2 6 \%}$ \\
\hline
\end{tabular}

Table 2 Mask type

\begin{tabular}{lll}
\hline Type of Mask & Number & Percentage \\
\hline N95 & $\mathbf{2 8 3}$ & $\mathbf{6 7 \%}$ \\
Surgical Mask & $\mathbf{1 4 0}$ & $\mathbf{3 3 \%}$ \\
\hline
\end{tabular}

Table 3 Sex

\begin{tabular}{lll}
\hline SEX & Number & Percentage \\
\hline Male & $\mathbf{3 2 0}$ & $\mathbf{7 6 \%}$ \\
Female & $\mathbf{1 0 3}$ & $\mathbf{2 4 \%}$ \\
\hline
\end{tabular}

Table 4 Symptoms

\begin{tabular}{lll}
\hline Symptoms & Number OF HCW & Percentage \\
\hline Headache & $\mathbf{9 7}$ & $\mathbf{2 3 \%}$ \\
Nasal Dryness & $\mathbf{9 4}$ & $\mathbf{2 2 \%}$ \\
Eye Dryness & $\mathbf{8 1}$ & $\mathbf{1 9 \%}$ \\
Acne & $\mathbf{5 1}$ & $\mathbf{1 2 \%}$ \\
Skin Breakdown & $\mathbf{2 1}$ & $\mathbf{5 \%}$ \\
Impaired Cognition & $\mathbf{1 8}$ & $\mathbf{5 \%}$ \\
Epistaxis & $\mathbf{2 2}$ & $\mathbf{5 \%}$ \\
None & $\mathbf{3 9}$ & $\mathbf{9 \%}$ \\
\hline
\end{tabular}

seen in the HCW who were wearing contact lenses. Acne and Skin Breakdown was also the problem mainly after 2 to $3 \mathrm{~h}$ of wearing maskfor which they have to consult the dermatologist for moisturizers and other topical medication which can be applied prior to wearing of mask. The most challenging part was Impaired Cognition although it was in few HCW after wearing the mask for mor than $1 \mathrm{~h}$ for that $\mathrm{HCW}$ used to removed the mask time to time for proper $\mathrm{O}_{2}$ inhalation and $\mathrm{CO}_{2}$ exhalation but it increases the transmission of COVID-19 infection.

\section{Discussion}

A profound number of $\mathrm{HCW}$ who participated in this survey reported adverse reactions to prolonged mask use during COVID-19. Headaches, skin breakdown, acne, and impaired cognition were all recognized as common adverse effects (Table 5). As we are still amidst the pandemic, and second waves are predicted in the future, tips and recommendations for enduring prolonged mask use are vital for the health and comfort of healthcare professionals. A hot and humid environment found in the facial region covered 
Table 5 No Previous History

\begin{tabular}{lll}
\hline No previous history & Number Of HCW & Percentage \\
\hline Headache & $\mathbf{2 4 5}$ & $\mathbf{5 8 \%}$ \\
Nasal Dryness & $\mathbf{3 8 7}$ & $\mathbf{9 1 \%}$ \\
Eye Dryness & $\mathbf{4 0 9}$ & $\mathbf{9 7 \%}$ \\
Acne & $\mathbf{2 2 3}$ & $\mathbf{5 3 \%}$ \\
Skin Breakdown & $\mathbf{3 8 3}$ & $\mathbf{9 0 \%}$ \\
Impaired Cognition & $\mathbf{4 2 1}$ & $\mathbf{9 9 \%}$ \\
Epistaxis & $\mathbf{4 0 3}$ & $\mathbf{9 5 \%}$ \\
\hline
\end{tabular}

by masks, causes discomfort and hyperthermia. This may create a situation where the $\mathrm{HCW}$ is unable to recognize dangers and perform manual tasks, and it also significantly affects motor skills. The moist environment and pressure from tight fitting masks also block facial ducts. This can explain the increase of acne with prolonged mask use. Frequent PPE and mask changes may cause shearing and breakdown of the skin, and breakdown on the bridge of the nose and cheek bones can be attributed to tight fitting masks and goggles that put pressure on these specific areas [4-6]. The first is frequent work breaks to be incorporated into work shifts to allow for shorter duration of mask use and reduction of PPE exposure. A second recommendation is preventative measures such as applying moisturizers, emollients, and barrier creams to prevent skin breakdown. Caution should be taken that dressings, moisturizers, and lotions do not interfere with the seal of the mask thereby causing decreased protection against COVID-19 particles. A third recommendation is improved mask design with a focus on safety, comfort, and tolerability $[1,7,8]$.

Headaches related to prolonged mask use can be attributed to mechanical factors, hypercapnia, and hypoxemia. Tight straps and pressure on superficial facial and cervical nerves are mechanical features causing headaches [1].

Tight fitting masks cause inadequate ventilation and increased levels of carbon dioxide (CO2) known as hypercapnia. As $\mathrm{CO} 2$ is a known respiratory stimulant, a buildup of exhaled $\mathrm{CO} 2$ between the mask and face will cause increased lung ventilation and respiratory activity. Symptoms of hypoxemia such as chest discomfort and tachypnea are also noted in healthcare professionals with prolonged mask use. Exhaled $\mathrm{CO} 2$ builds up between the mask and face, and increased levels of $\mathrm{CO} 2$ cause confusion, impaired cognition, and disorientation. To prevent headaches and impaired cognition, survey respondents recommend frequent short breaks, neck massage, increased hydration especially before start of the shift, alternating between surgical and N95 masks (if possible), and wearing a mask that fits one's face best [4].

For Eye dryness frequent use of eye lubricant to be done to prevent any further ulceration and in case of nasal dryness and to prevent epistaxis topical saline drops or gels to be used $[9,10]$.

\section{Limitations}

There are some limitations to this study. First, preexisting conditions such as high BMI, asthma, and other conditions were not assessed in this survey, and these could be impacting or increasing the adverse effects addressed in this survey. Second, issues such as stress level and quality sleep were also not included in this survey.

\section{Conclusion}

This study identified various adverse effects of prolonged mask use HCW during the COVID-19 pandemic. While $\mathrm{HCW}$ and the world at large wish this pandemic to end, and never return, various recommendations are presented for future prevention and management of these adverse effects.

To manage all the conditions as per study one should have to be proactive regarding the complications of prolong use of N95 mask and always carry their paraphernalia while moving for their long shifts in this COVID-19 pandemic.

\section{References}

1. Lim ECH, Seet RCS, Lee K-H, Wilder-Smith EPV, Chuah BYS et al (2006) Headaches and the N95 face-mask amongst healthcare providers. Acta Neurol Scand 113:199-202

2. Center for Disease Control and Prevention (2020) NIOSH-approved N95 particulate filtering facepiece respirators: Ancillary respirator information

3. Foo C, Goon ATJ, Leow Y-H, Goh C-L (2006) Adverse skin reactions to personal protective equipment against severe acute respiratory syndrome-a descriptive study in Singapore. Contact Dermatitis 55:291-294

4. Johnson AT (2016) Respirator masks protect health but impact performance: a review. J Biol Eng 10:4

5. Ong JJY, Bharatendu C, Goh Y, Tang JZY, Sooi KWX et al (2020) Headaches associated with personal protective equipmentA cross sectional study among frontline health- care workers during COVID-19. Headache 60:864-877

6. Lan J, Song Z, Miao X, Li H, Li Y et al (2020) Skin dam- age among health care workers managing coronavirus dis- ease-2019. J Am Acad Dermatol 82:1215-1216

7. Rebmann T, Carrico R, Wang J (2013) Physiologic and other effects and compliance with long-term respirator use among 
medical intensive care unit nurses. Am J Infect Control 41:1218-1223

8. Darlenski R, Tsankov N (2020) Covid-19 pandemic and the skinWhat should dermatologists know? Clin Dermatol 38:785-787

9. Moshirfar M, West Jr WB, Marx DP (2020) Face Mask-Associated Ocular Irritation and Dryness. Ophthalmol Ther 9:397-400
10. Zhu J, Lee S, Wang D (2014) Heow Pueh Lee. J Biosci Med 2:1-5

Publisher's Note Springer Nature remains neutral with regard to jurisdictional claims in published maps and institutional affiliations. 